\title{
The orbital motion of the Arches cluster: clues on cluster formation near the Galactic Center
}

\author{
Andrea Stolte - Andrea Ghez • Mark Morris • \\ Jessica Lu • Wolfgang Brandner · Keith Matthews
}

Received: 1 February 2008 / Accepted: 22 March 2008 / Published online: 3 November 2009

(C) Springer Science+Business Media B.V. 2009

\begin{abstract}
We have measured the proper motion of the Arches cluster near the Galactic Center (GC) with respect to the ambient field, using Keck/NIRC2 LGS-AO and VLT/NAOS-CONICA NGS-AO observations spanning a baseline of 4.3 yr. Combined with the radial velocity, we derive a 3D space motion of $232 \pm 30 \mathrm{~km} \mathrm{~s}^{-1}$ for the Arches cluster. This motion is exceptionally large compared to molecular cloud orbits at the GC, and places stringent constraints on the formation scenarios for starburst clusters in dense, nuclear environments.
\end{abstract}

Keywords Open clusters: individual: Arches · Galactic Center · Techniques: adaptive optics

\section{Introduction}

The Arches cluster is one of only three massive starburst clusters observed close to the Galactic Center (GC), at projected distances $<30 \mathrm{pc}$. With an age of only $2.5 \mathrm{Myr}$, it is in an almost pristine stage of cluster evolution, while its older twin, the Quintuplet, appears already dispersed at an age of $4 \mathrm{Myr}$, as a consequence of rapid dynamical evolution in the GC tidal field. The third known young, massive cluster is the central cluster located in the inner few pc around

A. Stolte $(\varangle) \cdot$ A. Ghez $\cdot$ M. Morris $\cdot$ J. Lu

University of California, Los Angeles, CA, USA

e-mail: stolte@astro.ucla.edu

W. Brandner

Max-Planck-Institute for Astronomy, Heidelberg, Germany

K. Matthews

California Institute of Technology, Pasadena, CA, USA the supermassive black hole (SMBH), which comprises stars with ages of $\sim 6 \mathrm{Myr}$ (e.g. Paumard et al. 2006). As the immediate environment around the SMBH is tidally heated and inhospitable to star formation (Morris 1993; Ghez et al. 2003, 2005), star clusters forming at larger radii have been suggested to spiral into the central few pc and replenish the young population. The in-spiral scenario has recently been strengthened by the detection of dense groups of young, co-moving stars in the central pc (Lu et al. 2005; Paumard et al. 2006). With the observation of the space motion of the Arches cluster, we can probe the in-spiral scenario for this cluster directly.

\section{Observations and geometric transformation}

Keck/NIRC2 laser-assisted adaptive optics $K^{\prime}$ observations taken in July 2006 were combined with VLT/NAOSCONICA (NACO) $H K_{\mathrm{S}}$ data obtained in March 2002. The final resolution on the combined image was diffractionlimited at the Keck 10-m dish, at 53 mas (FWHM) in the 18-min $K^{\prime}$ exposure, and a resolution of 84 mas (FWHM) was achieved in the 7-min NACO $K_{\mathrm{S}}$ frame. A detailed description of the data reduction and analysis can be found in Stolte et al. (2008). The geometric transformation between both images was fitted with a 2D second-order polynomial, derived from bright stars on the Arches main sequence, with a final astrometric mapping uncertainty of 2 mas (rms). Proper motions were then measured in this cluster reference frame over the $4.3 \mathrm{yr}$ baseline of the observations.

\section{The Arches cluster proper motion}

The proper motion diagram of stars in the central 10 arcsec of the Arches cluster core is shown in Fig. 1. Clus- 


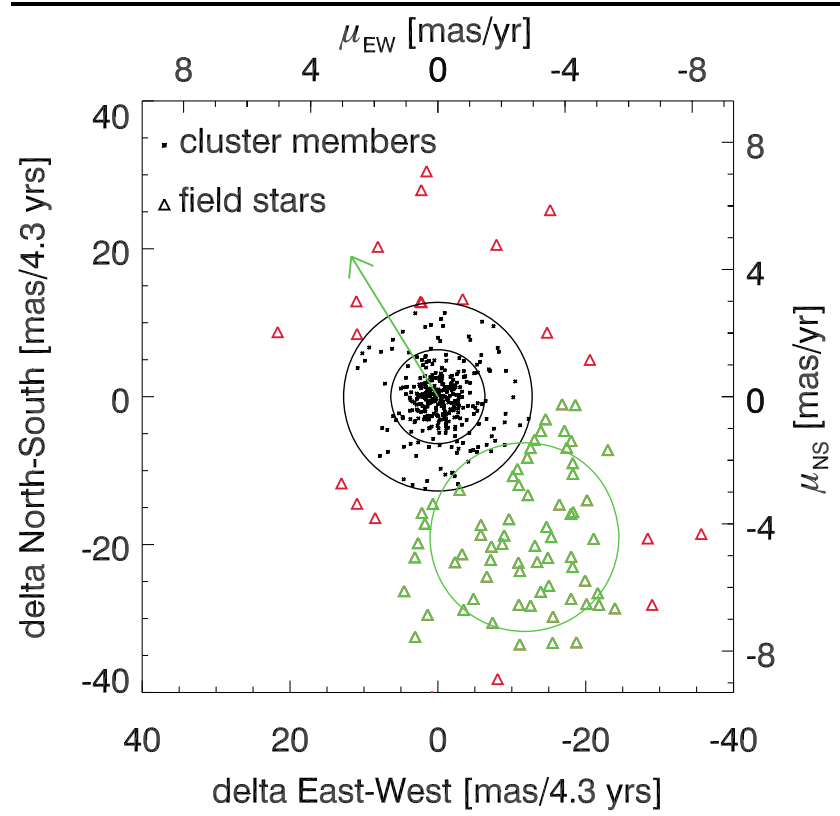

Fig. 1 Proper motion diagram of the Arches cluster. In the cluster reference frame, member candidates cluster around zero (dots), while

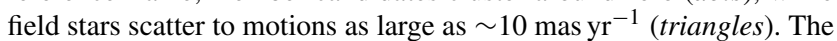
field population is dominated by relative motion to the south-west, from which the cluster proper motion relative to the ambient field is derived at $212 \pm 29 \mathrm{~km} \mathrm{~s}^{-1}$ to the north east and parallel to the Galactic plane

ter member candidates are clustering around zero motion in the cluster reference frame, while field stars scatter to

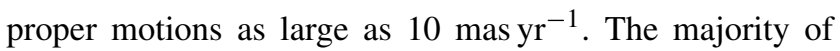
field stars, however, is found in the south-west quadrant in proper motion space. As the field population is dominated by bulge stars in the inner Galaxy, a random distribution of orbital velocities is expected along the line of sight. The clustering of field proper motions to the south-west in the cluster reference frame can therefore be interpreted as the proper motion of the Arches to the north-east with respect to the field. The proper motion of the Arches is measured from the mean of the field distribution in the southwest quadrant of the proper motion diagram at $212 \pm 29$ $\mathrm{km} \mathrm{s}^{-1}$.

A possible concern arises regarding the bias of field stars to the near side of the bulge. As the near side of the central bar rotates from west to east, field stars on the near side display predominantly eastward motion, in the opposite direction to the observed velocities, which decreases the apparent motion of the cluster relative to the field. Therefore, the derived proper motion of $212 \mathrm{~km} \mathrm{~s}^{-1}$ is a lower limit to the absolute cluster velocity. Combination with the radial velocity of $95 \pm 8 \mathrm{~km} \mathrm{~s}^{-1}$ (Figer et al. 2002) results in a 3D space motion of $232 \pm 30 \mathrm{~km} \mathrm{~s}^{-1}$ for the Arches cluster.

\section{Formation scenarios for the Arches cluster}

With a total stellar mass of $\sim 10^{4} \mathrm{M}_{\odot}$, the relaxation time of a GC starburst cluster at a distance of $30 \mathrm{pc}$ from the Center is on the order of a Gyr. With ages as young as a few Myr, the central starburst clusters should still move with the velocity inherited from their natal clouds. Clouds on stable orbits in the central molecular zone $\left(r_{\mathrm{GC}} \leq 200 \mathrm{pc}\right)$ are suggested to follow spheroidal $x 2$ orbits in the Milky Way bar potential (e.g. Binney et al. 1991; Englmaier and Gerhard 1999). Molecular clouds with sufficiently high densities to allow for fragmentation and the formation of a massive cluster display line-of-sight velocities of $v_{\text {los }}<120 \mathrm{~km} \mathrm{~s}^{-1}$ in radio CS surveys (Dame et al. 2001), in agreement with velocities of $x 2$ orbits in the bar model. These velocities are a factor of two too low to explain the high orbital motion of the Arches cluster. While the formation of the cluster is mysterious, the most likely trigger to form a high-velocity cluster appears to be a collision of two massive clouds (Stolte et al. 2008). Such a scenario was suggested as the triggering event for the burst of star formation in Sgr B2 (Hasegawa et al. 1994). However, this study suggests that the rate at which high-velocity clouds could migrate inwards from larger GC distances is not well constrained. Candidates for such a collision are clouds on $x 1$ orbits following the bar's major axis outside the $x 2$ (minor axis) orbital zone, as already suggested by Binney et al. (1991), or clouds falling in from the outer parts of the Galaxy (Crawford et al. 2002). A collision between an $x 1$ - and an $x 2$-orbit cloud is particularly intriguing, as $x 1$ clouds display line-of-sight velocities of up to $270 \mathrm{~km} \mathrm{~s}^{-1}$ (Dame et al. 2001), which could account for the orbital velocity of $232 \mathrm{~km} \mathrm{~s}^{-1}$ observed for the Arches cluster today. One particular problem with this scenario is that the cluster would need to inherit the high velocity of the infalling cloud, which requires a massive, high-density cloud capable to transfer its momentum to the natal cloud on the $x 2$ orbit. While this scenario would explain the observed velocity vectors of the Arches cluster's proper motion very well, molecular clouds on bar orbits outside the central molecular zone $\left(r_{\mathrm{GC}}>200 \mathrm{pc}\right)$ do not display highdensity material in present-day radio maps, and would therefore not be able to dominate the kinetic energy transfer during such a collision. This implies that either the colliding cloud does not originate from the pool of clouds on stable bar orbits, or that clouds with higher density were present at the time of the cluster's formation, 2.5 Myr ago but are not observed anymore at the present epoch. These caveats deepen the mystery of the formation of the Arches cluster.

\section{The orbital motion in the GC potential}

We have modeled the GC potential using logarithmic potential approximations (see Stolte et al. 2008 for details). 


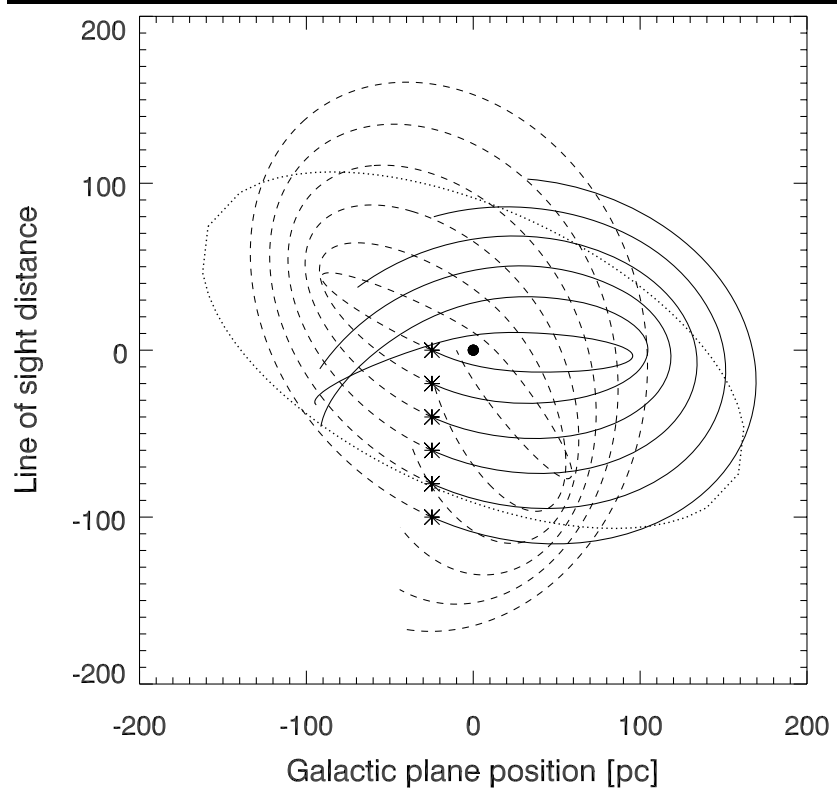

Fig. 2 Simulation of the Arches orbit in the inner Galaxy. The SMBH is located at the origin, and the projection represents the view from above the Galactic plane. The unconstrained line-of-sight distance of the cluster with respect to the GC is varied from -100 to $0 \mathrm{pc}$ in steps of $20 \mathrm{pc}$ (asterisks). The orbit is integrated for $2.5 \mathrm{Myr}$ backwards to the suspected origin of the Arches cluster (solid lines), and $360^{\circ}$ into the future (dashed lines). The dotted line approximates the location of the outer boundary of $x 2$ orbits in the bar (Bissantz et al. 2003), assuming a bar rotation angle of $25^{\circ}$ (Rattenbury et al. 2007). Note that for these orbits with galactocentric distances inside $\sim 100 \mathrm{pc}$, an origin near the $x 2-x 1$ orbital boundary (dotted line) is possible, while a cluster origin near the $x 2$ boundary becomes increasingly unlikely at larger galactocentric distances

The enclosed mass-radius relation was fitted by taking into account the gravitational potentials from the nuclear stellar cluster, the thick nuclear stellar disk, and the central black hole, as recently determined by Launhardt et al. (2002). Beyond the thick stellar disk, at $r_{\mathrm{GC}}>200 \mathrm{pc}$, the potential was smoothly transformed to the bar potential, which was approximated by a logarithmic potential as well, to ensure continuity in the test-particle acceleration. The Arches cluster was evolved forward and backward in time in this potential, with the aim to constrain the GC distance of the cluster's origin and the possibility for the Arches cluster to reach the inner few pc from the black hole.

The orbital simulations reveal that the Arches cluster can only reach the inner $10 \mathrm{pc}$ around the SMBH if its absolute GC distance is very close to its projected distance, i.e. if the cluster has a negligible line-of-sight radius component with respect to the GC. Even on the most radial orbit, the cluster will not come closer to the SMBH than $10 \mathrm{pc}$, and has also not been closer to the SMBH during its lifetime. This limits the amount of tidal stripping in the high-density environment of the nucleus, and implies that the Arches cluster does not contribute any young stars to the young stellar population near the SMBH. The observed 3D velocity of the cluster provides the first direct observational evidence that Archeslike clusters do not spiral into the GC during the lifetime of the massive stars, which is in excellent agreement with earlier $N$-body simulations based on assumptions related to the initial cluster mass and the radial velocity alone (e.g. Kim and Morris 2003).

As the Arches and Quintuplet clusters appear to not be sufficiently massive for the in-spiral case (Gerhard 2001; Kim and Morris 2003), we do not observe any progenitor clusters for the GC cluster at the present epoch. Nevertheless, it cannot be excluded that a substantially more massive young stellar cluster formed $6 \mathrm{Myr}$ ago in the inner few pc and migrated inwards, to provide the young stars observed in comoving groups in the central pc. Alternatively, the young stars could have formed in a massive thick disk, overcoming the tidal forces near the SMBH (Nayakshin et al. 2007). In any event, the observational constraint excluding Archeslike clusters as the source for young stars in the GC supports local star formation in the inner few pc around the SMBH.

\section{Conclusions}

The measurement of the 3D velocity of the Arches cluster enabled the detailed study of cluster formation scenarios in the central molecular zone, and allowed for the simulation of the cluster orbit in the GC potential. The orbital analysis suggests that the Arches is very unlikely to pass through the inner $10 \mathrm{pc}$ of the Galaxy, which implies that the cluster does not contribute young stars to the stellar population near the SMBH. If located inside the central molecular zone $\left(r_{\mathrm{GC}}<200 \mathrm{pc}\right)$, the orbital simulations suggest that the cluster completed between one quarter and at most one full orbit around the GC. The rapid motion of the cluster through the high-density environment near the GC might influence the tidal stripping of stars, and thus the dynamical evolution of the cluster mass. We therefore caution that the observed present-day mass function of the Arches cluster might not be the pristine initial stellar mass function at the time of cluster formation.

These findings should trigger a new set of dynamical simulations to constrain the evolution of the cluster population and the still mysterious origin of the Arches cluster.

Acknowledgements We would like to thank the organizers of the workshop for the invitation to attend and for the possibility to participate in the active discussions on massive cluster formation at this interesting meeting.

\section{References}

Bissantz, N., Englmaier, P., Gerhard, O.: Mon. Not. R. Astron. Soc. 340, 949 (2003) 
Binney, J., Gerhard, O.E., Stark, A.A., Bally, J., Uchida, K.I.: Mon. Not. R. Astron. Soc. 252, 210 (1991)

Crawford, I.A., Lallement, R., Price, R.J., Sfeir, D.M., Wakker, B.P., Welsh, B.Y.: Mon. Not. R. Astron. Soc. 337, 720 (2002)

Dame, T.M., Hartmann, D., Thadeus, P.: Astrophys. J. 547, 792 (2001)

Englmaier, P., Gerhard, O.: Mon. Not. R. Astron. Soc. 304, 512 (1999)

Figer, D.F., et al.: Astrophys. J. 581, 258 (2002)

Gerhard, O.: Astrophys. J. 546, 39 (2001)

Ghez, A.M., et al.: Astrophys. J. 586, 127 (2003)

Ghez, A.M., et al.: Astrophys. J. 620, 744 (2005)

Hasegawa, T., Fumio, S., Whiteoak, J.B., Miyawaki, R.: Astrophys. J. 429, 77 (1994)
Kim, S.S., Morris, M.: Astrophys. J. 597, 312 (2003)

Launhardt, R., Zylka, R., Mezger, P.G.: Astron. Astrophys. 384, 112 (2002)

Lu, J.R., Ghez, A.M., Hornstein, S.D., Morris, M., Becklin, E.E.: Astrophys. J. 625, 51 (2005)

Morris, M.: Astrophys. J. 408, 496 (1993)

Nayakshin, S., Cuadra, J., Springel, V.: Mon. Not. R. Astron. Soc. 379, 21 (2007)

Paumard, T., et al.: Astrophys. J. 643, 1011 (2006)

Rattenbury, N.J., Mao, S., Sumi, T., Smith, M.C.: Mon. Not. R. Astron. Soc. 378, 1064 (2007)

Stolte, A., et al.: Astrophys. J. 675, 1278 (2008) 